\title{
Editor's Welcome
}

\author{
ARVE ASBJØRNSEN \\ Lead Editor, University of Bergen, Norway
}

The Journal of Prison Education and Reentry is now entering its second year. The first issue of Volume 2 includes a wide variety of topics across countries and continents. The research articles cover topics of ADHD among prisoners, racial factors of post-release employment, and the perceived role of correctional staff in the reentry process. Asbjørnsen et al, discuss the signs of ADHD and how they might be related to education and work experience among incarcerated adults in Norway. They report how lack of education and former work experiences are closely related to increased signs of ADHD as they follow from the Utah approach to ADHD in the adults. This may have important consequences for planning and delivering educational services in prisons, as the need for bringing in the philosophy and knowledge from special and remedial teaching should be acknowledged. Lockwood et al. then discuss their study of employment and recidivism among individuals returning to the community from U.S. prisons, and show how patterns of employability and recidivism differ by race. Following a survey among 6349 released prisoners, they report that African American ex-prisoners had a higher unemployment rate and recidivism rate than Caucasian ex-prisoners. The results further revealed that released ex-prisoners, if employed, would likely be under-employed and experience difficulties in sustaining employment. And most important: post-release employment and level of education were the two most influential predictors of recidivism among ex-prisoners, regardless of ethnicity. Maybe the reentry process is a good place to elaborate some of the disparities that are also a result of ethnicity issues? Gunnison et al, share the results of their survey of 142 correctional practitioners. Although they achieved a low response rate (904 practitioners were invited to participate), their study reveals important information on the diverse ways custody staff perceive the prisoners and the centrality of their role in the reentry process. Housing and employment were topics raised by wardens and corrections officials as important needs for successful reentry. But it is also worth noticing that education does not seem to appear as a relevant topic in this discussion. Is this a finding that can be generalized, or is this particular for the US? We hope to see a discussion of this in a future issue.

The practitioner papers also include new and important insights from a diverse range of voices. Dreisinger contributes the second part of "Prisons, pedagogy, and pipelines" in which she shares her experiences establishing a college program in a prison in the US. Rausch provides a provocative paper, "Your True Freedom", describing some of her experiences in U.S. jails while teaching inmates "the fundamental truths of self worth, self acceptance and self love through writing, mindfulness meditation and emotional healing". This may not be "mainstream" classroom management everywhere in prison education, but the approach may be recognized in other self-management programs contributing to the reentry process in some countries? Simmons and Branch's paper on "servant leadership" describes a model for work in prison that is inspired by religious philosophical models, that may sound quite exotic to many practitioners in prison education, but the paper also explores a philosophical platform for establishing understanding of the process towards reentry. In addition, Jane Carrigan's paper presents an important and challenging discussion on the practice of doing research in prisons and on prison education. Carrigan's paper might be seen as a hybrid paper that spans both sections of our Journal in the voice of a research-practitioner discussing the practice of doing research.

We hope this issue challenges and stretches the reader in some way, generates fresh and creative ideas, or opens up the possibility of a new way of understanding. As well, we hope it inspires researchers and practitioners from all over the world to write, to submit manuscripts to JPER, and to share their wisdom with colleagues. The greater the range of cultures and systems represented in these pages, the deeper our individual and collective contributions will be to the advancement of prison education and reentry.

Correspondence: Arve Asbjørnsen, Email: arve.asbjornsen@psybp.uib.no

(Accepted 13 July 2015) ISSN: 2387-2306 doi: http://dx.doi.org/10.15845/jper.v2i2.833

Except where otherwise noted, content on this site is licensed under a Creative Commons Attribution 4.0 International License. 